\title{
BMJ Open Study protocol: a phase III randomised, double-blind, parallel arm, stratified, block randomised, placebo-controlled trial investigating the clinical effect and cost-effectiveness of sertraline for the palliative relief of breathlessness in people with chronic breathlessness
}

\author{
Gareth J Watts, ${ }^{1}$ Katherine Clark, ${ }^{1,2}$ Meera Agar, ${ }^{3,4,5,6}$ Patricia M Davidson, ${ }^{3,7}$ \\ Christine McDonald, ${ }^{8}$ Lawrence T Lam, ${ }^{3}$ Dimitar Sajkov, ${ }^{9}$ Nicola McCaffrey, ${ }^{5}$ \\ Matthew Doogue, ${ }^{10}$ Amy P Abernethy, ${ }^{11}$ David C Currow, ${ }^{5}$ On behalf of the \\ Australian national Palliative Care Clinical Studies Collaborative (PaCCSC)
}

To cite: Watts GJ, Clark K, Agar M, et al. Study protocol: a phase III randomised, double-blind, parallel arm, stratified, block randomised, placebo-controlled trial investigating the clinical effect and cost-effectiveness of sertraline for the palliative relief of breathlessness in people with chronic breathlessness. BMJ Open 2016;6: 013177.

doi:10.1136/bmjopen-2016013177

- Prepublication history for this paper is available online. To view these files please visit the journal online (http://dx.doi.org/10.1136/ bmjopen-2016-013177).

Received 24 June 2016 Revised 22 September 2016 Accepted 26 October 2016

CrossMark

For numbered affiliations see end of article.

Correspondence to Professor David C Currow; david.currow@sa.gov.au

\section{ABSTRACT}

Introduction: Breathlessness remains a highly prevalent and distressing symptom for many patients with progressive life-limiting illnesses. Evidence-based interventions for chronic breathlessness are limited, and there is an ongoing need for high-quality research into developing management strategies for optimal palliation of this complex symptom. Previous studies have suggested that selective serotonin reuptake inhibitors such as sertraline may have a role in reducing breathlessness. This paper presents the protocol for a large, adequately powered randomised study evaluating the use of sertraline for chronic breathlessness in people with progressive life-limiting illnesses. Methods and analysis: A total of 240 participants with modified Medical Research Council Dyspnoea Scale breathlessness of level 2 or higher will be randomised to receive either sertraline or placebo for 28 days in this multisite, double-blind study. The dose will be titrated up every 3 days to a maximum of $100 \mathrm{mg}$ daily. The primary outcome will be to compare the efficacy of sertraline with placebo in relieving the intensity of worst breathlessness as assessed by a 0-100 mm Visual Analogue Scale. A number of other outcome measures and descriptors of breathlessness as well as caregiver assessments will also be recorded to ensure adequate analysis of participant breathlessness and to allow an economic analysis to be performed. Participants will also be given the option of continuing blinded treatment until either study data collection is complete or net benefit ceases.

Appropriate statistical analysis of primary and secondary outcomes will be used to describe the wealth of data obtained.

Ethics and dissemination: Ethics approval was obtained at all participating sites. Results of the study

\section{Strengths and limitations of this study}

- This is an adequately powered study to provide a clinically meaningful outcome.

- To optimise the generalisability of the findings, this multisite study will capture people from across a spectrum of care settings, including direct inpatient care, inpatient consultations, clinic attendances and community care.

- This study builds on the experience of several double-blind randomised controlled trials in therapeutic interventions for breathlessness.

- This is a relatively long study for participants from palliative care which may potentially influence completion rates independently of the intervention.

- The study has no end point assessing potential changes in function (eg, accelerometry) as a result of the intervention.

will be submitted for publication in peer-reviewed journals and the key findings presented at national and international conferences.

Trial registration number: ACTRN12610000464066.

\section{INTRODUCTION}

Increasing numbers of individuals are living with multiple chronic conditions and high symptom burdens. Internationally, policymakers are calling for therapies that focus on promoting not just the quantity but quality of life. ${ }^{12}$ Breathlessness is one of the most 
prevalent and distressing symptoms experienced by people with progressive life-limiting illness and is defined as "a subjective experience of breathing discomfort that consists of qualitatively distinct sensations that vary in intensity". ${ }^{3}$ The understanding of the physiological mechanisms as well as the interdependence of psychological, emotional, behavioural and environmental factors influencing the individual's experience of breathlessness has increased in recent years. ${ }^{4}$ There is an ongoing need for high-quality research into interventions that may help ensure optimal palliation of this distressing symptom. ${ }^{5}$

Chronic breathlessness is defined as persistent breathlessness at rest, or on minimal exertion despite optimal management of the underlying causes. ${ }^{6}{ }^{7}$ It affects $50-70 \%$ of people with advanced malignancy ${ }^{8} 9$ and an even higher proportion of those with end-stage respiratory or cardiac failure. ${ }^{10}$ In the later phases of progressive life-limiting illnesses breathlessness, unlike most other symptoms, typically worsens ${ }^{11}{ }^{12}$ and is a greatly feared symptom. ${ }^{13}$ This poses particular challenges for clinicians involved in providing symptom control and end of life care.

In spite of the prevalence and burden of breathlessness, management options are limited and the current evidence-based supports use of non-pharmacological interventions such as pulmonary rehabilitation and mobility aids; ${ }^{14}{ }^{15}$ oxygen therapy in those with evidence of hypoxia ${ }^{1617}$ and the potential of systemic, oral or parenteral morphine. ${ }^{6}{ }^{18-21}$ The complex interplay between physiological and psychosocial antecedents to the subjective experience of breathlessness justifies exploration of novel pharmacotherapeutic interventions. ${ }^{22} 23$

Serotonin as a neurotransmitter is strongly implicated in the control and regulation of respiratory physiology in the central nervous system. ${ }^{24}$ Sertraline is a selective serotonin reuptake inhibitor (SSRI) used widely as an antidepressant and in generalised anxiety disorders. A previously published literature review ${ }^{20}$ identified two non-randomised pilot studies ${ }^{25} 26$ and a case series ${ }^{27}$ that report symptomatic benefit from SSRIs when used to manage chronic breathlessness in patients with chronic obstructive pulmonary disease (COPD). Papp et $a l^{25}$ report a subjective improvement in well-being in six people with COPD, three of whom had a diagnosis of either phobia or panic disorder by standard psychiatric interview. These observations are supported by work by Smoller et $a l^{27}$ who reported a decrease in breathlessness and a subjective improvement in exercise tolerance in a case series of seven people treated with sertraline $25-100 \mathrm{mg}$ daily, three of whom met Diagnostic and Statistical Manual of Mental Disorders IV (DSM-IV) criteria for mood or anxiety disorders on structured clinical interview. Similar effects were found by Perna $e t a l^{26}$ in a small study looking at the use of citalopram for relief of breathlessness in six people, none of whom had a history of mood or anxiety disorders by DSM-IV criteria, reporting decreased breathing discomfort. Additionally, some studies which have investigated the use of SSRIs to manage anxiety and depression in the COPD population report that breathlessness scores are improved by the concomitant administration of these medications. ${ }^{28} 29$ These studies were small pilot studies and therefore not amenable to meta-analysis, ${ }^{30}$ however these data suggest a potential role for SSRIs in reducing the subjective sensation of breathlessness in people with chronic breathlessness even in the absence of anxiety or panic disorders.

This paper describes the protocol and presents the rationale for an adequately powered, multisite, stratified, block randomised, double-blind, parallel arm, placebocontrolled, titrated dose study investigating the role that sertraline has in the relief of chronic breathlessness. This phase III study aims to improve the evidence base for the pharmacological management of chronic breathlessness and forms part of a programme seeking to explore the role of existing medications in symptom control. This paper complies with the SPIRIT recommendations $^{31} 32$ for protocol reporting and has been adapted for publication from the original complete study protocol written by the Palliative Care Clinical Studies Collaborative (PaCCSC). ${ }^{33}$ The study will report against CONSORT guidelines. ${ }^{34} 35$

\section{METHODS AND ANALYSIS}

\section{Study design}

A placebo-controlled study was chosen because there is no registered medication for the pharmacological treatment of chronic breathlessness, and there is clinical equipoise regarding the net benefit of SSRIs in this clinical setting.

A 20 participant phase II study was initially conducted at one site to establish feasibility and acceptability of the proposed phase III study and to estimate the variance between the two groups. The phase II study was analysed while maintaining blinding of investigators and participants. There were no protocol changes following recruitment of these first 20 participants. Consequently, the phase III study proceeded to multisite recruitment, retaining these participants as the study design and procedures are identical.

Following informed consent and eligibility, participants will be randomised to receive either a daily dose of sertraline or matched placebo. The dose will be titrated in the first 7 days and continued for a total of 28 days (figure 1). If the participant perceives benefit from the intervention, they will be given the option of continuing the blinded treatment in an extension phase either until study data collection is complete or participants perceive that the net benefit ceases.

Participants will have assessments at baseline day 0, day 9 and day 29 (following primary end point completion on day 28) and day 35 (at the end of dose downward titration 6 days after treatment dose cessation) if the participant does not enter the extension phase. 
Figure 1 Study design diagram. VAS, Visual Analogue Scale.

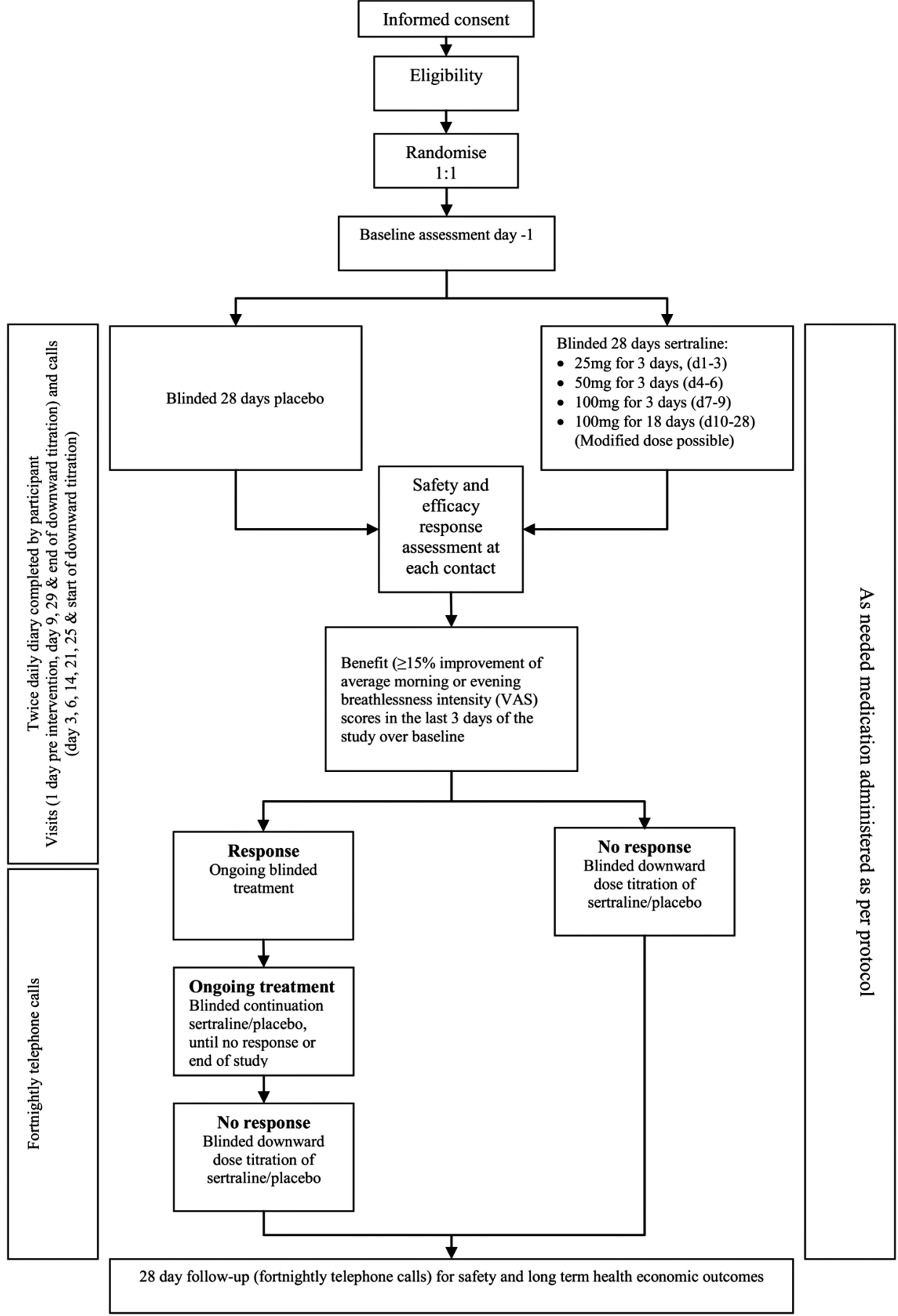

Regular telephone contact will also be made to assess safety and compliance.

If a participant agrees to enter the extension treatment phase of the study, they will receive fortnightly telephone contacts and monthly visits from a member of the research team. This will help to ensure ongoing, highquality data collection and encourage compliance with the intervention. Additionally, all participants will receive two fortnightly telephone calls following treatment cessation to collect data for the economic analysis and to continuously assess participant safety and adverse effects.

\section{Collaborating organisations}

This multisite study will be coordinated by the Australian national PaCCSC and sponsored by Flinders University, Adelaide, Australia. It will recruit participants from across Australia with the collaborating sites indicated in box 1. Clinical teams involved include respiratory medicine, cardiology, oncology, general medicine and palliative care. This diversity of clinical teams reflects the heterogeneity and pervasiveness of breathlessness across multiple chronic, progressive illnesses. 


\section{Box 1 List of collaborating sites}

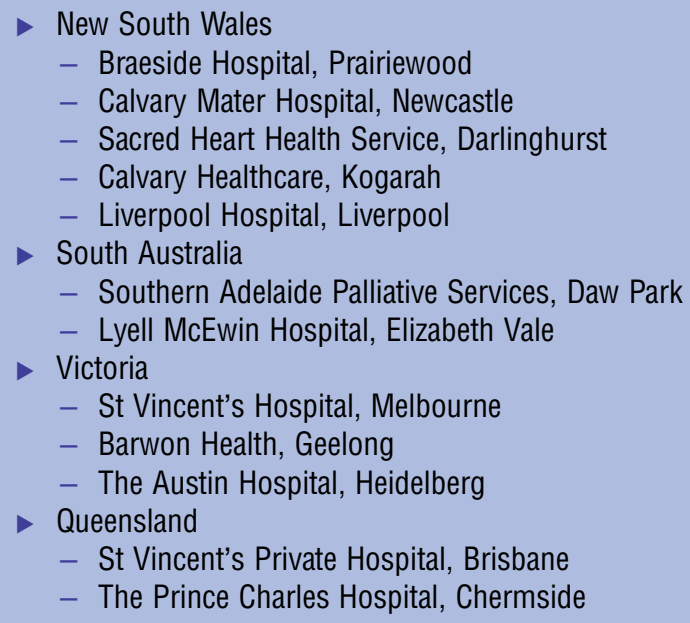

\section{Study objectives}

The primary objective of the study is to compare the efficacy of sertraline with placebo in relieving the intensity of worst breathlessness ${ }^{36}$ in the previous 12 hours as assessed by a $0-100 \mathrm{~mm}$ Visual Analogue Scale (VAS).

Secondary objectives include:

- Assessment of participant and caregiver quality of life and participant performance status.

- Assessment of the mastery subscale of the Chronic Respiratory Questionnaire (CRQ) between groups.

- Documentation and comparison of the side effects and frequency of side effects experienced in each arm.

- To determine which participants derive the greatest benefit from sertraline using data from baseline participant characteristics.

- Assessment of frequency of occurrence of common toxicities and adverse effects such as nausea and falls.

- Assessment of changes in anxiety and depression scores over the study period.

- Assessment of net effect (benefit vs harms).

- Economic analysis of the net effect of sertraline incremental to usual care from participant-level data collected on costs and effects.

\section{Study population}

Adults with breathlessness defined as level 2 or higher on the modified Medical Research Council (mMRC) Dyspnoea Scale $^{37}$ (table 1) despite optimal treatment of the underlying causes of breathlessness will be approached by study investigators.

Level 2 or higher breathlessness on this mMRC Scale corresponds with moderate-to-severe breathlessness. ${ }^{38}$ In addition to being over the age of 18 years and being able to speak and read English the inclusion criteria are:

- Chronic breathlessness where the underlying causes have been maximally treated confirmed by a specialist relevant to the potential participant's most significant underlying cause. The diagnosis of chronic is made once all identified reversible causes of breathlessness
Table 1 The modified Medical Research Council Dyspnoea Scale ${ }^{37}$

\section{Grade Description of breathlessness}

$0 \quad$ I only get breathless with strenuous exercise.

$1 \quad$ I get short of breath when hurrying on level ground or walking up a slight hill.

2 On level ground, I walk slower than people of the same age because of breathlessness, or I have to stop for breath when walking at my own pace on the level.

3 I stop for breath after walking about 100 yards or after a few minutes on level ground.

$4 \quad$ I am too breathless to leave the house or I am breathless when dressing.

are documented and optimally managed. There is no minimum duration for this diagnosis.

- No changes in medication for management of breathlessness for 1 week, except 'as needed' medications.

- Estimated life expectancy of at least 2 months.

- Be able to complete the informed consent process and consent to participate in the study.

Owing to the multiple possible reported adverse effects associated with sertraline and other SSRIs, a number of exclusion criteria will be applied:

- Previous adverse reaction to sertraline.

- Severe hepatic impairment defined as Child-Pugh ${ }^{39}$ Class C or higher.

- Gastrointestinal bleeding within the previous 6 months.

- Plasma sodium of $<128 \mathrm{mmol} / \mathrm{L}$.

- Recent difficult seizure control.

- Respiratory depression with a respiratory rate $<8$ breaths per minute or previous opioid-induced respiratory failure.

- A respiratory or cardiac event in the previous week (not including mild upper respiratory tract infections).

- Pregnant or breast feeding.

- Depressive symptoms as defined by Hospital Anxiety and Depression (HADS) ${ }^{40}$ Subscale $>16$.

- Current treatment with medicines that increase the risk of serotonin syndrome (box 2).

\section{Recruitment and consent}

Potentially eligible participants will be identified by their treating clinicians and referred to the local research team. Case identification in relevant outpatient clinics and inpatient services using case note review will be undertaken, with permission to approach potentially eligible participants given by the relevant case manager or consultant.

Written informed consent to participate in this study will follow a process of information exchange between study staff, clinicians and potential participants and caregivers. Participant information sheets will form the basis 
Box 2 Examples of medicines ${ }^{41}$ that increase the risk of serotonin syndrome

Examples of medicines with increased risk of serotonin syndrome

Prescribed medications

- Selective serotonin reuptake inhibitors (SSRI)

- Citalopram, escitalopram, paroxetine, fluoxetine, clomipramine

- Serotonin-norepinephrine reuptake inhibitors (SNRI)

- Venlafaxine

- Noradrenergic and specific serotonergic antidepressant (NaSSA)

- Mirtazapine

- Tricyclic antidepressants (TCA)

- Amitriptyline

- Serotonin receptor agonists

- Buspirone, triptans

- Monoamine oxidase inhibitors (MAOI)

- Moclobemide (within 2 days of entry into study)

- Phenelzine, tranylcypromine (within 4 weeks of entry to study)

- Amphetamine-type drugs

- Dexamphetamine, methylphenidate

Weight-loss medication

- Phenteramine, diethylpropion, sibutramine

Other prescription medications

- Lithium, carbamazepine, linezolid, imatinib

Over-the-counter medication

- Dextromethorphan, chlorpheniramine, brompheniramine

Complementary therapies

- St John's Wort, tryptophan, S-adenosyl-methionine

of discussion, and the participant will be given as much time as necessary to consider the study. Site coordinators and research nurses at each involved site are trained in the consent procedures for this study.

Any participant who consents to be included in the study may also nominate their primary caregiver (or person likely to provide care if they were to need care at some time in the future) for involvement in the study. The caregiver will be asked to consent to completion of a caregiver-specific quality of life assessment to be included in the study. Consent by caregivers to complete this assessment will not affect participant eligibility.

\section{Randomisation}

At each site involved, on referral to the study, participants will be sequentially allocated a unique identifying number to be used for all subsequent study documentation. This will ensure confidentiality is maintained. The randomisation request will be generated by the receipt of a prescription of the study drugs by a clinical trial site pharmacist. Randomisation will be stratified according to a baseline measure of the anxiety and depression subscales of the HADS. ${ }^{40}$ Initial stratification will be into two groups according to HADS anxiety subscale; $0-10$ (normal to mild) or 11-21 (moderate to severe). Within these two groups, participants will be further stratified into two further groups categorised by HADS depression
Table 2 Randomisation strata according to HADS subscale scores ${ }^{40}$

\begin{tabular}{lllll}
\hline & Stratum & Stratum & Stratum & Stratum \\
& $\mathbf{1}$ & $\mathbf{2}$ & $\mathbf{3}$ & $\mathbf{4}$ \\
\hline HADS Anxiety & $0-10$ & $11-21$ & $0-10$ & $11-21$ \\
Subscale Score & & & & \\
HADS & $0-10$ & $0-10$ & $11-16$ & $11-16$ \\
$\begin{array}{l}\text { Depression } \\
\text { Subscale Score }\end{array}$ & & & & \\
\hline $\begin{array}{l}\text { HADS, Hospital Anxiety and Depression Scale. } \\
\text { HAl }\end{array}$
\end{tabular}

subscale; 0-10 (normal to mild) or 11-16 (moderate). This will create four strata (table 2).

Within each site and within each of the four strata, randomisation will occur in blocks of four in a 1:1 ratio to ensure even allocation.

\section{Intervention}

All participants will take one blinded opaque capsule each morning on waking for 28 days. Participants and investigators will be blinded to the medication provided. As there is a theoretical concern that SSRIs can blunt carbon dioxide sensitivity ${ }^{25} 42$ and because SSRIs are rarely tolerated at full dose when initiated without titration, therapy will be initiated at low dose with titration over 7 days. Those in the active arm will start oral sertraline $25 \mathrm{mg}$ daily (figure 1). This dose will then double every 3 days to reach a maximum dose of sertraline $100 \mathrm{mg}$ daily by the beginning of day 7 if tolerated. Those in the control arm will receive an identical appearing dose of placebo titrated over the same period of time. Low-dose oral morphine has demonstrated benefit over placebo for symptomatic relief of chronic breathlessness, ${ }^{6} 1921{ }^{43}$ hence, all participants will be prescribed immediate release morphine solution. Participants will be permitted to take $2.5 \mathrm{mg}$ of oral morphine immediate release solution every 4 hours up to six doses per day on an as needed basis. Docusate with senna will also be prescribed to all participants for treatment of opioid-induced constipation if required.

Participation in the study will cease if adverse effects related to the study medicines develop and are unacceptable to the participant or clinician in charge of the participant's care. Adverse effects will be classified by National Cancer Institute Common Terminology Criteria for Adverse Events, V.4 (NCI CTC AE v4), ${ }^{44}$ recorded and treated symptomatically. Anyone with NCI CTC AE v4 scores of 3 not responding to symptomatic treatment or scores of 4 or higher will be withdrawn from the study. Other reasons for discontinuation of study medicines are if treatment is deemed ineffective by the treating clinician and an alternative therapy is required, increasing breathlessness despite study medication and maximal use of rescue morphine solution. Withdrawal of consent, hyponatraemia, falls and other adverse events may also cause withdrawal from the study. Reasons for withdrawal will be documented. 
Participants will be monitored for harms at days 3, 6 and 9. If adverse effects persist despite symptomatic treatment, participants may continue to take part in the study but will continue at the sertraline dose prior to adverse symptoms being noted. If adverse effects are noted at the starting dose of sertraline $25 \mathrm{mg}$, participants will be withdrawn from the study.

Assessment of net clinical benefit will be conducted on day 29 of the study by study nurses for the primary outcome of worst breathlessness averaged over the last three study days and the adverse effects of the intervention that were specifically sought during the study. If there is no clinical benefit, dose will be tapered down by halving the dose every 3 days to $25 \mathrm{mg}$ and then stopped. As previously mentioned, participants will have the option of continuing the blinded treatment until completion of study data collection or benefit ceases.

\section{Data collection and outcome measures}

Current literature suggests that there may be two discrete pathways involved in the sensation of breathlessness. O'Donnell et al suggest that a two-phase gating model for breathlessness exists. A first gate considers the intensity of breathlessness; the second considers its unpleasantness. ${ }^{45}$ Some studies suggest that people can discriminate between these two pathways. ${ }^{11} 46$ Participants in this study will therefore be asked to rate breathlessness intensity and unpleasantness using VAS and Likert Scales (box 3).

Changes in breathlessness intensity will be measured on a $100 \mathrm{~mm}$ VAS and 4-point Likert Scales (box 3). Participants will be asked to complete a diary in the morning and evening at baseline and on days 7, 14, 26, 27 and 28 (table 3), recording the intensity and unpleasantness of breathlessness using the two scales. The primary outcome measure will be based on average worst morning and evening breathlessness intensity based on VAS scores on days 26, 27 and 28 of the intervention period. A $15 \%$ improvement in breathlessness score from baseline is considered a clinically meaningful improvement, consistent with patient-defined minimally clinically important differences in VAS Scales. ${ }^{47}$

Secondary outcomes will be assessed by completion of a number of validated participant and research teammediated measures at various time points throughout the study period. Box 3 gives an overview of the questionnaires and scales used in this study, and table 3 provides an overview of the study timeline and the outcome measures recorded at each time point. The range of assessment tools will ensure quantitative and qualitative assessment of participants' symptom severity and ensure measurement of quality of life for participants and caregivers in line with Australian National Palliative Care Strategy's aims. ${ }^{48}$

Blood sampling will be required for laboratory assessment of full blood count, electrolytes, liver and renal function at eligibility. A sample will be taken at eligibility assessment if no sample is available from the preceding
7 days. Child-Pugh scoring of hepatic dysfunction ${ }^{39}$ will also occur at eligibility. Sertraline is known to cause hyponatraemia so serum sodium levels will be monitored weekly throughout the study.

Respiratory and cardiac function will be recorded from recent spirometry and echocardiography testing if available but are not essential for participation in this study. Owing to the theoretical risk of respiratory depression, end-tidal carbon dioxide levels and pulse oximetry will be measured at baseline and at study exit.

Demographics including age, gender, availability of primary caregiver, postcode, ability to read and speak English and Aboriginal or Torres Strait Islander status will be recorded. In addition, main clinical diagnoses will be detailed including the dominant aetiology of the breathlessness, co-morbid illnesses and smoking history recorded in pack years. The Charlson Comorbidity Index (CCI) will be used as a uniform measure of co-morbidity and tested as an independent predictor of long-term survival. ${ }^{62} 63$

The participant diary will form an important source of data collection. It is completed at baseline and on days 7 , 14, 21 and on the past 3 days of intervention 26, 27 and 28 (table 3). It will be used to document and record:

- Breathlessness intensity and unpleasantness VAS and Likert Scales.

- Current, average and worst breathlessness and relief of breathlessness using VAS and Likert Scales.

- mMRC breathlessness scale measures functional impairment due to breathlessness. ${ }^{37} 52$

- Symptoms associated with sertraline use using Likert Scales.

- Well-being and global quality of life using numerical rating scale.

- Record of 'as needed' medication usage.

Data will be collected on potential adverse effects of sertraline including nausea; diarrhoea; tremor; agitation and restlessness; sexual dysfunction and general wellbeing. Adverse events are assessed according to Good Clinical Practice (GCP) guidelines ${ }^{64}$ and recorded and reported according to PaCCSC standard operating procedures.

Data will also be collected from participants on the use of hospital services, specialised palliative care services, community nurses, local General Practitioner/ practice nurses and changes in medicines to inform the economic analysis.

\section{Sample size calculation}

As previously mentioned, this is a phase III study that incorporates a blinded analysis of 20 participants from a single-site phase II study with an identical protocol. This analysis was conducted and overseen by the Data Safety Monitoring Committee. The blinded results were shared with the study investigators to ensure feasibility, acceptability of the outcome measures and that the variance of the primary outcome measure was within the anticipated range. No changes were made to the phase III protocol 
Box 3 Assessment methods and questionnaires used in this study

Intensity of breathlessness

- Visual Analogue Scale (VAS)

- $100 \mathrm{~mm}$ scale

- $0=$ ='no breathlessness at all'; 100='breathlessness as bad as you can imagine'

- Likert Scale

- 4-point scale: none, mild, moderate and severe

Unpleasantness of breathlessness

$\checkmark$ VAS

- $100 \mathrm{~mm}$ scale

- $0=$ 'not unpleasant at all'; $100=$ 'the most unpleasant breathlessness l've ever felt'

- Likert Scale

- 4-point scale: none, mild, moderate, severe

Descriptors of breathlessness ${ }^{49-51}$

- Fifteen categorical descriptors presented to participants in random order

- Participants choose appropriate descriptors that characterise their breathlessness and indicate up to three most applicable statements

Modified Medical Research Council (mMRC) Dyspnoea Scale ${ }^{37} 52$

- 5-point (0-4) categorical breathlessness scale

- Descriptive measure of functional impairment due to breathlessness

- Lower scores imply less breathlessness

Chronic Respiratory Questionnaire (CRQ) -Dyspnoea Subscale 5354

- Total of 20 questions covering social and emotional symptoms and perceptions of breathlessness in relation to five activities over the preceding 2 weeks

Higher scores imply better respiratory function

Global impression of change

7-point scale regarding participant perception of change since starting study

- From 'very much worse' to 'very much improved'

- Adapted for measurement of breathlessness from original ${ }^{55}$

- Higher scores imply better global quality of life

Life space assessment ${ }^{56}$

- Yes or no responses to simple questions assessing mobility including where a person goes, frequency and dependency when mobilising

- Higher scores imply better mobility

Australia-modified Karnofsky Performance Status (AKPS) ${ }^{57}$

- Validated variant of Karnofsky performance status

- Scored 0-100 in increments of 10 assigned to participants based on ability to perform activities of daily living

- Higher scores imply better level of function

Hospital Anxiety and Depression Scale (HADS) ${ }^{40}$

- 14-item questionnaire consisting of two 7-item subscales looking at depression and anxiety, respectively

- Higher scores are associated with greater morbidity

Folstein Mini-Mental Status Examination (MMSE) ${ }^{58}$

11-task examination assessing higher cognitive function scored out of 30

- Higher scores suggest better executive functioning

EORTC QLQ-C15 (European Organization for Research and Treatment of Cancer-Quality of Life Questionnaire 15)

15-question subset of the original 30 question assessment of health-related quality of life ${ }^{59}$

- Higher scores suggest better quality of life

CQOLC (Caregiver Quality of Life Index) ${ }^{60}$

-Well-established quality of life assessment for caregivers of people with cancer ${ }^{61}$

35-item questionnaire assessing physical, social, emotional, financial aspects of well-being

- Higher scores suggest better quality of life

as a result of this analysis. Using these data, 75 completed participants in each study group will provide $\sim 80 \%$ power at a two-tailed type 1 error of 0.05 to detect a difference of $41 \%$ of participants in the sertraline group meeting the definition of response, versus $20 \%$ in the placebo group. The study needs to recruit 60 participants in each stratum randomised 1:1 between intervention and control. Allowing for attrition, 240 people will be randomised to give 150 complete sets of data.

\section{Statistical analysis}

The null hypothesis of the study is that there is no difference between the net clinical effects of sertraline and placebo on the sensation of chronic breathlessness. As previously detailed, response is defined as at least $15 \%$ improvement in breathlessness scores from baseline.

Analysis of the primary outcome will be through comparison of the percentages of participants achieving a $15 \%$ reduction in breathlessness from baseline in the two study groups by $\chi^{2}$ testing without continuity 
Table 3 Table of study measures according to time point and timeline

\begin{tabular}{|c|c|c|c|c|c|c|c|c|c|c|c|c|c|c|c|c|}
\hline \multirow[b]{2}{*}{$\begin{array}{l}\text { Time point } \\
\text { Assessment type } \\
\text { Timeline day }\end{array}$} & \multirow[b]{2}{*}{ Eligibility } & \multicolumn{5}{|c|}{ Dose up-titration and core study period } & \multicolumn{2}{|c|}{$\begin{array}{l}\text { Dose } \\
\text { down-titration }\end{array}$} & \multirow[b]{2}{*}{$\begin{array}{l}\text { Treatment } \\
\text { cessation }\end{array}$} & \multicolumn{6}{|c|}{ Ongoing treatment phase } & \multirow{2}{*}{$\begin{array}{l}\text { Follow-up } \\
\text { Fortnightly } \\
\text { for } 4 \text { weeks }\end{array}$} \\
\hline & & $\begin{array}{c}-1 \\
\text { Baseline } \\
0\end{array}$ & $\begin{array}{l}0 \text { and } 1 \\
\text { T/C } \\
3 \text { and } 6\end{array}$ & $\begin{array}{c}2 \\
\text { Visit } \\
9\end{array}$ & $\begin{array}{c}3,4,5 \\
\text { T/C } \\
14,21,25\end{array}$ & $\begin{array}{c}6 \\
\text { Visit } \\
29^{\star}\end{array}$ & $\begin{array}{c}7 \\
\text { T/C } \\
\text { End +3 }\end{array}$ & $\begin{array}{c}8 \\
\text { Visit } \\
\text { End+6 }\end{array}$ & & $\begin{array}{l}1 \\
\mathrm{~T} / \mathrm{C} \\
42\end{array}$ & $\begin{array}{c}2 \\
\text { Visit } \\
56\end{array}$ & $\begin{array}{c}3 \\
T / C \\
70\end{array}$ & $\begin{array}{c}4 \\
\text { Visit } \\
84\end{array}$ & $\begin{array}{c}5 \\
T / C \\
98\end{array}$ & $\begin{array}{c}6 \\
\text { Visit } \\
112\end{array}$ & \\
\hline \multicolumn{17}{|c|}{ Research team mediated assessments } \\
\hline Vital signs & & $\checkmark$ & & $\checkmark$ & & $\checkmark$ & & $\checkmark$ & $\checkmark$ & & $\checkmark$ & & $\checkmark$ & & $\checkmark$ & \\
\hline $\begin{array}{l}\text { Bloods: FBC, Elec, CC } \\
\text { Child-Pugh }\end{array}$ & $\begin{array}{l}\checkmark \\
\checkmark\end{array}$ & & & & & & & & & & & & & & & \\
\hline $\begin{array}{l}\text { Child-Pugh } \\
\text { Pulse oximetry }\end{array}$ & $\checkmark$ & & & & & $\checkmark$ & & & $\checkmark$ & & $\checkmark$ & & $\checkmark$ & & $\checkmark$ & \\
\hline Spirometry & $\checkmark$ & & & & & $\checkmark$ & & & $\checkmark$ & & & & & & 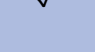 & \\
\hline Echocardiography & $\checkmark$ & & & & & $\checkmark$ & & & $\checkmark$ & & & & & & & \\
\hline Serum sodium & $\checkmark$ & & $\checkmark$ & $\checkmark$ & $\checkmark$ & $\checkmark$ & $\checkmark$ & & $\checkmark$ & $\checkmark$ & $\checkmark$ & $\checkmark$ & $\checkmark$ & $\checkmark$ & $\checkmark$ & \\
\hline End-tidal $\mathrm{CO}_{2}$ & $\checkmark$ & & & & & $\checkmark$ & & & $\checkmark$ & & $\checkmark$ & & $\checkmark$ & & $\checkmark$ & \\
\hline Demographics & $\checkmark$ & $\checkmark$ & & & & & & & & & & & & & & \\
\hline $\begin{array}{l}\text { Medical and physical } \\
\text { examination }\end{array}$ & $\checkmark$ & & & & & & & & & & & & & & & \\
\hline Clinical history & $\checkmark$ & & & & & & & & & & & & & & & \\
\hline $\mathrm{CCl}$ & $\checkmark$ & & & & & & & & & & & & & & & \\
\hline AKPS & & $\checkmark$ & & $\checkmark$ & $\checkmark$ & $\checkmark$ & & & $\checkmark$ & & $\checkmark$ & & $\checkmark$ & & $\checkmark$ & \\
\hline Safety and $\mathrm{AE}$ & & $\checkmark$ & $\checkmark$ & $\checkmark$ & $\checkmark$ & $\checkmark$ & $\checkmark$ & $\checkmark$ & $\checkmark$ & $\checkmark$ & $\checkmark$ & $\checkmark$ & $\checkmark$ & $\checkmark$ & $\checkmark$ & $\checkmark$ \\
\hline Drug compliance & & & $\checkmark$ & $\checkmark$ & $\checkmark$ & $\checkmark$ & $\checkmark$ & $\checkmark$ & $\checkmark$ & $\checkmark$ & $\checkmark$ & $\checkmark$ & $\checkmark$ & $\checkmark$ & $\checkmark$ & \\
\hline $\begin{array}{l}\text { Global impression of } \\
\text { change }\end{array}$ & & & & & & $\checkmark$ & & & $\checkmark$ & & & & & & & \\
\hline $\begin{array}{l}\text { Desire to continue } \\
\text { therapy }\end{array}$ & & & & & & $\checkmark$ & & & $\checkmark$ & & & & & & & \\
\hline Economic analysis data & & & & & & & & & $\checkmark$ & & & & & & & $\checkmark$ \\
\hline Diary reminder & & & $\checkmark$ & & $\checkmark$ & & $\checkmark$ & & & & & & & & & \\
\hline \multicolumn{17}{|c|}{ Participant mediated assessments } \\
\hline MMSE & $\checkmark$ & & & & & & & & & & & & & & & \\
\hline $\begin{array}{l}\text { Descriptors of } \\
\text { breathlessness }\end{array}$ & & $\checkmark$ & & & & $\checkmark$ & & & $\checkmark$ & & & & & & & \\
\hline $\begin{array}{l}\text { mMRC Breathlessness } \\
\text { Scale }\end{array}$ & $\checkmark$ & & & & & & & & & & & & & & & \\
\hline $\begin{array}{l}\text { CRQ-Breathlessness } \\
\text { Scale }\end{array}$ & & $\checkmark$ & & & & $\checkmark$ & & & $\checkmark$ & & $\checkmark$ & & $\checkmark$ & & $\checkmark$ & $\checkmark$ \\
\hline EORTC QLQ-C15 & & $\checkmark$ & & $\checkmark$ & & $\checkmark$ & & & $\checkmark$ & & $\checkmark$ & & $\checkmark$ & & $\checkmark$ & $\checkmark$ \\
\hline CQOLC & & $\checkmark$ & & & & $\checkmark$ & & & $\checkmark$ & & & & & & & \\
\hline HADS & $\checkmark$ & $\checkmark$ & & & & $\checkmark$ & & & $\checkmark$ & & & & & & & \\
\hline Life space assessment & & $\checkmark$ & & $\checkmark$ & & $\checkmark$ & & & $\checkmark$ & & & & & & & \\
\hline
\end{tabular}




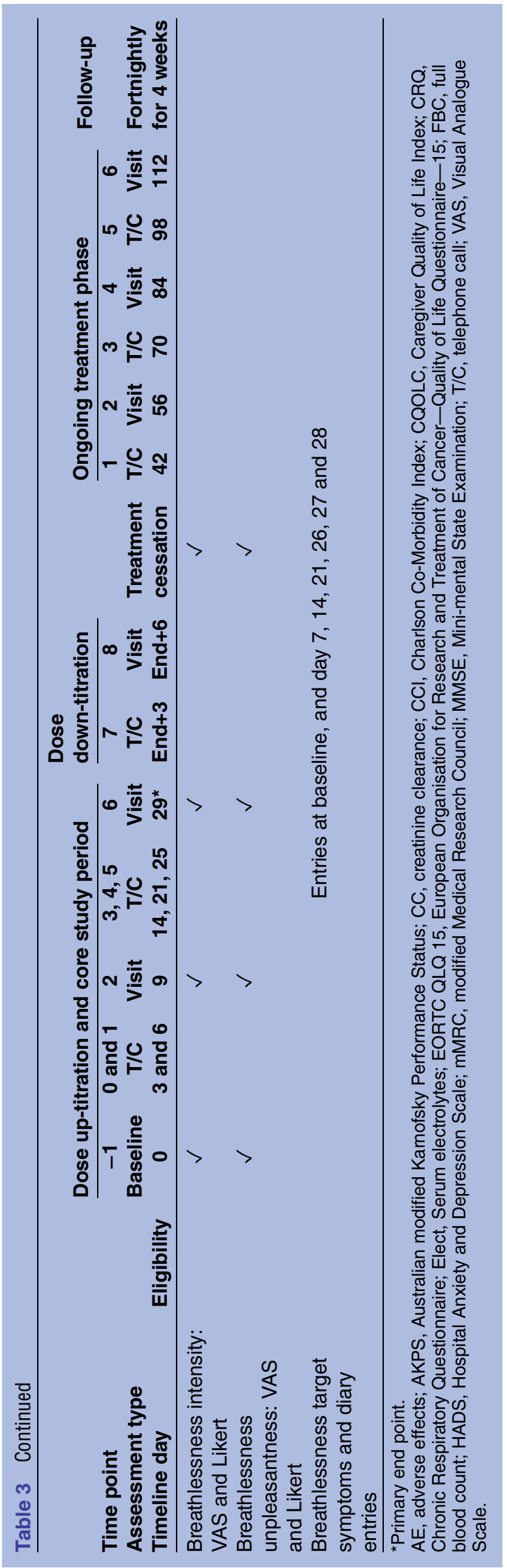

correction. Secondary analysis will be through longitudinal repeated measures mixed models with unstructured covariance matrices assessing group by time and group by intervention effects across the entire study period with particular focus on the past 3 days to compare each arm. This approach will be applied for breathlessness and Quality of Life (QOL) assessments, as well as main anticipated adverse effects.

Multivariable regression models will be used to establish clinically relevant baseline predictors of response (percent reduction over baseline breathlessness) exploring baseline breathlessness, baseline gas exchange, CCI, ${ }^{62}$ HADS scores ${ }^{40}$ including depression and anxiety subscores and functional status.

Secondary outcomes such as caregiver well-being, changes in functional status using Australian modified Karnofsky Performance Status (AKPS) ${ }^{57}$ (primarily as a safety measure) and utility associated with health quality of life between the placebo group and the sertraline group will be examined using Mann-Whitney $\mathrm{U}$ tests. This same statistical method will also be used to compare adverse effects between study arms. Missing data for primary and secondary end points will be dealt with by multiple imputations.

\section{Economic evaluation}

The main objective of the economic evaluation is to estimate the incremental costs, effects and cost-effectiveness of sertraline relative to placebo for the palliative relief of breathlessness in people with chronic breathlessness. A within-trial cost-effectiveness analysis will be undertaken from a healthcare provider perspective using participantlevel data collected from randomisation up to 28 days after completing the study intervention or death, whichever occurs sooner. Mean costs and effectiveness will be estimated for the sertraline and placebo arms including: sensation of breathlessness, quality of life, caregiver quality of life, intervention costs, hospitalisations, community support and other medications controlling for baseline use. Resource use will be costed according to the Australian Manual of Resource Items and their Associated Costs ${ }^{65}$ in Australian dollars. Incremental net monetary benefit (INMB) and cost-effectiveness acceptability curves (CEACs) will be estimated at potential threshold values for: (1) a $1 \%$ reduction in breathlessness and (2) one extra responder (response is defined as at least $15 \%$ improvement in breathlessness scores from baseline). INMB is the monetary value of additional effects of care minus the additional costs of care. ${ }^{66}$ CEACs graphically represent the probability of a positive INMB over plausible threshold values for the outcome of interest. The threshold value indicates the decisionmaker's maximum acceptable monetary value for 1 unit gain of effect. ${ }^{66}{ }^{67}$ Bootstrapping on participants' costs and effects across 5000 replicates will be used to assess the uncertainty for costs, effects and cost-effectiveness, and $95 \%$ CIs will be calculated for the INMB and CEACs using these replicates. ${ }^{68}$ 


\section{Ethical considerations}

In order to maximise the collection of valuable data while minimising patient burden, only outcome measures previously validated in this patient population will be collected. Invasive measures have been minimised to cause the least possible physical stress. It is recognised that completion of written study measures can be a significant source of stress and burden to the population who will participate in this study. Sensitive issues regarding the measurement of functional status can be confronting for participants and discussing quality of life and other questions may also cause psychological distress. As such, any emotional distress caused by the study would prompt intervention and support from the specialist palliative care team who would be involved in the care of the people participating in this study. The research team will always be attuned to monitoring for signs of participant distress, and ongoing training will be provided to the research teams in conjunction with GCP principles. $^{64}$

Consent to participate will be obtained by a research team member not involved in the participant's usual clinical care. This will also assist with the separation of research and clinical responsibilities of the clinical staff. Everyone has the ability to decline to participate in the study, and participants can withdraw at any time without detriment to the provision or quality of their clinical care.

The protocol has been initially reviewed and approved by the Cancer Institute New South Wales (CI NSW), Human Research Ethics Committee (HREC), New South Wales in 2010 (approval number 2010C/02/120) but was transferred to the Prince of Wales Hospital HREC in 2013 (approval number 13/327HREC/13/ POWH/785) following closure of the CI NSW HREC. Each individual collaborating site has also each obtained relevant HREC approvals to recruit to this study.

\section{Confidentiality}

Data will be retained in accordance with the principles of GCP. ${ }^{64}$ Participants will be allocated a unique identification (ID) number at entry. The master list linking participant personal information and ID number will be maintained in a separate locked cabinet and passwordprotected hard drive. Data will be analysed by ID number only. Records will be retained for 15 years after study completion and then destroyed in accordance with PaCCSC standard operating procedures consistent with current HREC requirements.

\section{Dissemination}

The results of this trial will be submitted for publication in peer-reviewed publications and the key findings presented at national and international conferences. If the study shows a net benefit, contact will also be made with key professional groups, and regulatory and funding bodies.

\section{DISCUSSION}

This study protocol represents a large, randomised study evaluating a non-opioid pharmacological intervention for chronic breathlessness in people with progressive lifelimiting illnesses. Chronic breathlessness is one of the most feared symptoms and the potential implications for quality of life are substantial if effective treatment can be found. Currently, there is no medicine registered internationally for the treatment of chronic breathlessness despite its prevalence. This study will evaluate a new, but theoretically useful therapy by quantifying net clinical effect as well as assessing cost-efficacy to inform clinicians, policymakers and patients.

The recently published randomised controlled trial investigating the use of the azapirone serotonergic anxiolytic buspirone $20 \mathrm{mg}$ daily in the management of cancer-related breathlessness is an example of the highquality studies that are now being undertaken. This large, multicentre phase II trial failed to show improvement in breathlessness as measured by the Oxygen Cost Diagram or anxiety, measured by the Spielberger State-Trait Anxiety Inventory, after 6 weeks of intervention. ${ }^{23}$ This study protocol continues the theme of investigating the potential uses of non-sedating anxiolytics in the management of breathlessness and builds on previous work by using a different medicine with careful dose titration in a larger and more diverse participant population for a longer follow-up period.

With the exception of buspirone, developing the evidence base to date has focused on morphine for the treatment of chronic breathlessness. Although the balance of evidence favours symptomatic benefit safety, many practitioners are still concerned about the potentially rare but catastrophic problem of respiratory depression when using opioids. The largest trials to date have sought to further minimise any risk by using regular, low dose oral extended release morphine to minimise peak drug concentrations. ${ }^{6} 1943$

Recruitment to randomised controlled palliative care studies is challenging but feasible if it is approached systematically. ${ }^{69}$ PaCCSC has a strong track record of successfully designing and conducting phase III studies in this patient population, ${ }^{71-73}$ making this the world's largest palliative care clinical trial group having randomised more than 1700 participants to phase III studies. The key to the anticipated success of this study is that it is being conducted across multiple sites which will allow adequate and timely recruitment. The trial began enrolling participants in January 2011 and as of April 2016 has randomised 192 participants with a projected recruitment completion date of March 2017. In addition, this protocol has been adapted by a group in the UK investigating the effect of mirtazapine in the management of chronic breathlessness in a similar group of participants. $^{74}$

The economic analysis will aid evidence-based decision-making in palliative care service provision and inform any subsequent regulatory or funding body 
applications by providing prospectively collected, and jointly considered data on health resource use and costs in the clinical setting of palliating chronic breathlessness.

\section{Author affiliations}

${ }^{1}$ Department of Palliative Care, Calvary Mater Newcastle, Newcastle, New South Wales, Australia

${ }^{2}$ School of Medicine and Public Health, The University of Newcastle, Newcastle, New South Wales, Australia

${ }^{3}$ Faculty of Health and Graduate School of Health, University of Technology Sydney, Sydney, New South Wales, Australia

${ }^{4}$ Clinical Trials, Ingham Institute of Applied Medical Research, Sydney, New South Wales, Australia

${ }^{5}$ Department of Palliative and Supportive Services, Flinders University, Adelaide, South Australia, Australia

${ }^{6}$ South West Sydney Clinical School, University of New South Wales, Sydney, New South Wales, Australia

${ }^{7}$ School of Nursing, Johns Hopkins University, Baltimore, Maryland, USA

${ }^{8}$ Austin Health, Respiratory and Sleep Medicine, Austin Hospital, Heidelberg, Victoria, Australia

${ }^{9}$ Australian Respiratory and Sleep Medicine Institute, Flinders Medical Centre, Adelaide, South Australia, Australia

${ }^{10}$ Department of Clinical Pharmacology, University of Otago, Christchurch, New Zealand

${ }^{11}$ Department of Medicine, Duke University Medical Center, Durham, North Carolina, USA

Collaborators All authors on behalf of the Australian national Palliative Care Clinical Studies Collaborative (PaCCSC).

Contributors GJW updated the literature review and wrote the manuscript by adapting the original study protocol for publication, subsequent reviews and amendments. KC contributed to the development and refining of the protocol, writing of manuscript and subsequent review. MA contributed to the development of the study protocol, writing of manuscript and subsequent review, and PMD contributed to the writing of the original study protocol and subsequent review. LL reviewed, commented and informed the methodology and statistical analytical approach of the protocol. CM, DS, MD and APA reviewed, commented and informed methodology, development and writing of the protocol. NM contributed to the conception and design of the study protocol specifically the design of the economic evaluation and participated in drafting the article and revising it critically for important intellectual content. DCC is the lead study investigator and lead author of original study protocol.

Funding This work was supported by the Australian Government, Commonwealth Department of Health through the National Palliative Care Program and NHMRC Project Grant (grant number GNT1010065) and sponsored by Flinders University.

Competing interests DCC has received an unrestricted research grant from Mundipharma, is an unpaid member of an advisory board for Helsinn Pharmaceuticals and has consulted to Mayne Pharma and received intellectual property payments from them.

Ethics approval Cancer Institute New South Wales (CI NSW), Human Research Ethics Committee (HREC), New South Wales in 2010 (approval number 2010C/02/120). Transferred to the Prince of Wales Hospital HREC in 2013 (approval number 13/327HREC/13/POWH/785).

Provenance and peer review Not commissioned; externally peer reviewed.

Data sharing statement No additional data are available.

Open Access This is an Open Access article distributed in accordance with the Creative Commons Attribution Non Commercial (CC BY-NC 4.0) license, which permits others to distribute, remix, adapt, build upon this work noncommercially, and license their derivative works on different terms, provided the original work is properly cited and the use is non-commercial. See: http:// creativecommons.org/licenses/by-nc/4.0/

\section{REFERENCES}

1. Worldwide Palliative Care Alliance. Global atlas of palliative care at the end of life-World Health Organization. London, UK: Worldwide Palliative Care Alliance, 2014. http://www.thewhpca.org/resources/ global-atlas-on-end-of-life-care

2. Higginson IJ. Evidence based palliative care: there is some evidence -and there needs to be more. BMJ 1999;319:462-3.

3. American Thoracic Society. Dyspnea: mechanisms, assessment, and management. A consensus statement. Am J Respir Crit Care Med 1999;159:321-40.

4. Parshall MB, Schwartzstein RM, Adams L, et al. An official American Thoracic Society statement: update on the mechanisms, assessment, and management of dyspnea. Am J Respir Crit Care Med 2012;185:435-52.

5. Currow DC, Abernethy AP, Allcroft P, et al. The need to research refractory breathlessness. Eur Resp J 2016;47:342-3.

6. Abernethy AP, Currow DC, Frith $\mathrm{P}$, et al. Randomised, double blind, placebo controlled crossover trial of sustained release morphine for the management of refractory dyspnoea. BMJ 2003;327:523-9.

7. Booth S, Bausewein C, Higginson I, et al. Pharmacological treatment of refractory breathlessness. Expert Rev Respir Med 2009;3:21-36.

8. Reuben DB, Mor V. Dyspnea in terminally ill cancer patients. Chest 1986;89:234-6.

9. Bruera E, Schmitz B, Pither J, et al. The frequency and correlates of dyspnea in patients with advanced cancer. J Pain Symptom Manage 2000;19:357-62.

10. Edmonds $\mathrm{P}$, Karlsen $\mathrm{S}$, Khan S, et al. A comparison of the palliative care needs of patients dying from chronic respiratory diseases and lung cancer. Palliat Med 2001;15:287-95.

11. Mercadante S, Casuccio A, Fulfaro F. The course of symptom frequency and intensity in advanced cancer patients followed at home. J Pain Symptom Manage 2000;20:104-12.

12. Ripamonti C, Fusco F. Respiratory problems in advanced cancer. Support Care Cancer 2002;10:204-16.

13. O'Driscoll M, Corner J, Bailey C. The experience of breathlessness in lung cancer. Eur J Cancer Care 1999;8:37-43.

14. Bausewein C, Booth S, Gysels M, et al. Non-pharmacological interventions for breathlessness in advanced stages of malignant and non-malignant diseases. Cochrane Database Syst Rev 2008; (2):CD005623.

15. McCarthy B, Casey D, Devane D, et al. Pulmonary rehabilitation for chronic obstructive pulmonary disease. Cochrane Database Syst Rev 2015;(2):CD003793.

16. Abernethy AP, McDonald CF, Frith PA, et al. Effect of palliative oxygen versus room air in relief of breathlessness in patients with refractory dyspnoea: a double-blind, randomised controlled trial. Lancet 2010;376:784-93.

17. Uronis HE, Ekstrom MP, Currow DC, et al. Oxygen for relief of dyspnoea in people with chronic obstructive pulmonary disease who would not qualify for home oxygen: a systematic review and meta-analysis. Thorax 2015;70:492-4.

18. Johnson MJ, Bland JM, Oxberry SG, et al. Opioids for chronic refractory breathlessness: patient predictors of beneficial response. Eur Resp J 2013;42:758-66.

19. Ekstrom M, Nilsson F, Abernethy AA, et al. Effects of opioids on breathlessness and exercise capacity in chronic obstructive pulmonary disease. A systematic review. Ann Am Thorac Soc 2015;12:1079-92.

20. Uronis HE, Currow DC, Abernethy AP. Palliative management of refractory dyspnea in COPD. Int J Chron Obstruct Pulmon Dis 2006;1:289-304.

21. Jennings AL, Davies AN, Higgins JP, et al. A systematic review of the use of opioids in the management of dyspnoea. Thorax 2002;57:939-44.

22. Currow DC, Abernethy AP. Pharmacological management of dyspnoea. Curr Opin Support Palliat Care 2007;1:96-101.

23. Peoples AR, Bushunow PW, Garland SN, et al. Buspirone for management of dyspnea in cancer patients receiving chemotherapy: a randomized placebo-controlled URCC CCOP study. Support Care Cancer 2016;24:1339-47.

24. Bonham AC. Neurotransmitters in the CNS control of breathing Respir Physiol 1995;101:219-30.

25. Papp LA, Weiss JR, Greenberg HE, et al. Sertraline for chronic obstructive pulmonary disease and comorbid anxiety and mood disorders. Am J Psychiatry 1995;152:1531.

26. Perna G, Cogo R, Bellodi L. Selective serotonin re-uptake inhibitors beyond psychiatry: are they useful in the treatment of severe, chronic obstructive pulmonary disease? Depress Anxiety 2004;20:203-4.

27. Smoller JW, Pollack MH, Systrom D, et al. Sertraline effects on dyspnea in patients with obstructive airways disease. Psychosomatics 1998;39:24-9. 
28. Lacasse $Y$, Beaudoin L, Rousseau L, et al. Randomized trial of paroxetine in end-stage COPD. Monaldi Arch Chest Dis 2004;61:140-7.

29. Eiser N, Harte R, Spiros K, et al. Effect of treating depression on quality-of-life and exercise tolerance in severe COPD. COPD 2005;2:233-41.

30. Usmani ZA, Carson KV, Cheng JN, et al. Pharmacological interventions for the treatment of anxiety disorders in chronic obstructive pulmonary disease. Cochrane Database Syst Rev 2011; Issue 11:CD008483. doi: 10.1002/14651858.CD008483.pub2

31. Chan AW, Tetzlaff JM, Altman DG, et al. SPIRIT 2013 statement: defining standard protocol items for clinical trials. Ann Intern Med 2013:158:200-7.

32. Chan AW, Tetzlaff JM, Gotzsche PC, et al. SPIRIT 2013 explanation and elaboration: guidance for protocols of clinical trials. $B M J$ 2013;346:e7586.

33. Currow DC, McDonald C, Plummer J, et al. Randomised doubleblind mulit-site parallel arm controlled trial to assess relief of refractory breathlessness comparing oral sertraline and placebo. Version 013/09 V4.3. Palliat Care Clin Stud Collabrat. (In press).

34. Schulz KF, Altman DG, Moher D. CONSORT 2010 Statement: updated guidelines for reporting parallel group randomised trials. BMJ 2010;340:c332.

35. Moher D, Hopewell S, Schulz KF, et al. CONSORT 2010 Explanation and Elaboration: updated guidelines for reporting parallel group randomised trials. J Clin Epidemiol 2010;63:e1-37.

36. Johnson MJ, Close L, Gillon SC, et al. Use of the modified Borg Scale and numerical rating scale to measure chronic breathlessness: a pooled data analysis. Eur Respir J 2016;47:1861-4.

37. Mahler DA, Wells CK. Evaluation of clinical methods for rating dyspnea. Chest 1988;93:580-6.

38. Wedzicha JA, Bestall JC, Garrod R, et al. Randomized controlled trial of pulmonary rehabilitation in severe chronic obstructive pulmonary disease patients, stratified with the MRC Dyspnoea Scale. Eur Respir J 1998;12:363-9.

39. Pugh RN, Murray-Lyon IM, Dawson JL, et al. Transection of the oesophagus for bleeding oesophageal varices. Br J Surg 1973;60:646-9.

40. Zigmond AS, Snaith RP. The Hospital Anxiety and Depression Scale. Acta Psychiatr Scand 1983;67:361-70.

41. Australian medicines handbook. Adelaide: Australian Medicines Handbook Pty, 2015; https://shop.amh.net.au/faq/58.

42. Henderson DR, Konkle DM, Mitchell GS. Effects of serotonin re-uptake inhibition on ventilatory control in goats. Respir Physiol 1999:115:1-10.

43. Currow DC, McDonald C, Oaten S, et al. Once-daily opioids for chronic dyspnea: a dose increment and pharmacovigilance study. J Pain Symptom Manage 2011;42:388-99.

44. National Cancer Institute. Common Terminology Criteria for Adverse Events (CTCAE) Version v4.0. 2009; May 29, 2009.

45. O'Donnell DE, Banzett RB, Carrieri-Kohlman V, et al. Pathophysiology of dyspnea in chronic obstructive pulmonary disease: a roundtable. Proc Am Thorac Soc 2007:4:145-68.

46. von Leupoldt A, Dahme B. Differentiation between the sensory and affective dimension of dyspnea during resistive load breathing in normal subjects. Chest 2005;128:3345-9.

47. Riles AL. Minimally clinically important difference for the UCSD shortness of breath questionnaire, Borg Scale and Visual Analog Scale. COPD 2005;2:105-10.

48. Care CDoHaA. National Palliative Care Strategy: A National Framework for Palliative Care Service Development. Publications Production Unit (Public Affairs, Parliamentary and Access Branch) Publications approval number 4065, 2000.

49. Simon PM, Schwartzstein RM, Weiss JW, et al. Distinguishable types of dyspnea in patients with shortness of breath. Am Rev Respir Dis 1990;142:1009-14.

50. Wilcock A, Crosby V, Hughes A, et al. Descriptors of breathlessness in patients with cancer and other cardiorespiratory diseases. $J$ Pain Symptom Manage 2002;23:182-90.

51. Harver A, Mahler DA, Schwartzstein RM, et al. Descriptors of breathlessness in healthy individuals. Chest 2000;118:679-90.

52. Bestall JC, Paul EA, Garrod R, et al. Usefulness of the Medical Research Council (MRC) Dyspnoea Scale as a measure of disability in patients with chronic obstructive pulmonary disease. Thorax 1999;54:581-6.

53. Puhan MA, Behnke M, Frey M, et al. Self-administration and interviewer-administration of the German Chronic Respiratory
Questionnaire: instrument development and assessment of validity and reliability in two randomised studies. Health Qual Life Outcomes 2004;2:1

54. Schunemann HJ, Guyatt GH, Griffith L, et al. A randomized controlled trial to evaluate the effect of informing patients about their pretreatment responses to two respiratory questionnaires. Chest 2002;122:1701-8.

55. Farrar JT, Young JPJr, LaMoreaux L, et al. Clinical importance of changes in chronic pain intensity measured on an 11-point numerical pain rating scale. Pain 2001;94:149-58

56. Brown CJ, Roth DL, Allman RM, et al. Trajectories of life-space mobility after hospitalization. Ann Intern Med 2009;150:372-8.

57. Abernethy AP, Shelby-James T, Fazekas BS, et al. The Australia-modified Karnofsky Performance Status (AKPS) Scale: a revised scale for contemporary palliative care clinical practice [ISRCTN81117481]. BMC Palliat Care 2005;4:7.

58. Folstein MF, Folstein SE, McHugh PR. "Mini-mental state". A practical method for grading the cognitive state of patients for the clinician. J Psychiatr Res 1975;12:189-98.

59. Aaronson NK, Ahmedzai S, Bergman B, et al. The European Organization for Research and Treatment of Cancer QLQ-C30 a quality-of-life instrument for use in international clinical trials in oncology. J Natl Cancer Inst 1993;85:365-76.

60. Weitzner MA, Jacobsen PB, Wagner $\mathrm{H}$ Jr, et al. The Caregiver Quality of Life Index-Cancer (CQOLC) Scale: development and validation of an instrument to measure quality of life of the family caregiver of patients with cancer. Qual Life Res 1999;8:55-63.

61. Edwards B, Ung L. Quality of life instruments for caregivers of patients with cancer: a review of their psychometric properties. Cancer Nurs 2002;25:342-9.

62. Charlson ME, Pompei P, Ales KL, et al. A new method of classifying prognostic comorbidity in longitudinal studies: development and validation. J Chronic Dis 1987;40:373-83.

63. Degroot V, Beckerman H, Lankhorst G, et al. How to measure comorbidity: a critical review of available methods. J Clin Epidemiol 2003;56:221-9.

64. Group IEW. ICH Harmonised Tripartite Guideline: Guideline for Good Clinical Practice. International Conference on Harmonisation of Technical Requirements for Registration of Pharmaceuticals for Human Use; 1996. http://www.ich.org/fileadmin/Public_Web_Site/ ICH_Products/Guidelines/Efficacy/E6/E6_R1_Guideline.pdf (accessed 23 Jun 2016).

65. Australian Government, Department of Health and Ageing. Manual of resource items and their associated costs-December 2009. Canberra, ACT, 2009. http://www.pbs.gov.au/info/industry/ useful-resources/manual

66. Stinnett AA, Mullahy J. Net health benefits: a new framework for the analysis of uncertainty in cost-effectiveness analysis. Med Decis Making 1998;18(2 Suppl):S68-80.

67. Fenwick E, Byford S. A guide to cost-effectiveness acceptability curves. Br J Psychiatry 2005;187:106-8.

68. Campbell MK, Torgerson DJ. Bootstrapping: estimating confidence intervals for cost-effectiveness ratios. $Q J \mathrm{Med}$ 1999;92:177-82.

69. LeBlanc TW, Lodato JE, Currow DC, et al. Overcoming recruitment challenges in palliative care clinical trials. $J$ Oncol Pract 2013;9:277-82.

70. Hanson LC, Bull J, Wessell K, et al. Strategies to support recruitment of patients with Life-limiting illness for research: the Palliative Care Research Cooperative Group. J Pain Symptom Manage 2014:48:1021-30

71. Hardy J, Quinn S, Fazekas B, et al. Randomized, double-blind, placebo-controlled study to assess the efficacy and toxicity of subcutaneous ketamine in the management of cancer pain. J Clin Oncol 2012;30:3611-17.

72. Currow DC, Quinn S, Agar M, et al. Double-blind, placebo-controlled, randomized trial of octreotide in malignant bowel obstruction. J Pain Symptom Manage 2015;49:814-21.

73. Agar M, Lawlor P, Quinn S, et al. Phase III randomised double-blind controlled trial of oral risperidone, haloperidol or placebo with rescue subcutaneous midazolam for delirium management in palliative care. Australas J Ageing 2015;34:33.

74. EU Clinical Trials Register. BETTER-B (Feasibility) BETter TreatmEnts for Refractory Breathlessness: a feasibility study of the use of mirtazapine for refractory breathlessness. UK: King's College London, 2016. EudraCT Number: 2015-004064-11. http://www. clinicaltrialsregister.eu 